\title{
XV.
}

\section{Ueber die Wirkung der Oxalsäure und einiger ihrer Derivate auf die Nieren.}

(Aus der medicinischen Universitätsłlinik zu Göttingen.)

Experimentelle Untersuchungen

von Wilhelm Ebstein und Arthur Nicolaier.

(Hierzu Taf. V.)

Die nachstehenden Untersuchungen wurden zunächst unternommen, um mittelst Einverleibung gewisser Stoffwechselprodukte und ihrer Derivate in den thierischen Organismus auf experimentellem Wege Harnsteine zu erzeugen. Die in dieser Beziehung mit Oxamid angesteliten Untersuchungen haben zu einem positiven Ergebniss geführt und sind von uns bereits an anderen Orten veröffentlicht worden. Wir werden uns daher begnügen, über diesen Gegenstand einige ergänzende Bemerkungen hier mitzutheilen. Im Wesentlichen ist der Zweck nachstehender Erörterungen die Mittheilung der Versuche, welche betreffs der Steinbildung zu einem negativen Resultate führten, dagegen in anderer Richtung mancherlei nicht uninteressante Aufschlüsse gaben. Das Fragmentarische einzelner dieser Versuchsreihen möge damit entschuldigt werden, dass sie ursprünglich einen anderen $Z$ weck verfolgten und abgebrochen wurden, als sie in dieser Beziehung orgebnisslos verliefen. Wir haben trotzdem diese Versuche kurz mitgetheilt, weil sie vielleicht Anderen Veranlassung zur weiteren Verfolgung des Gegenstandes bieten werden.

Es handelt sich hier wesentlich darum, zu ermitteln, welchen Einfluss die Einverleibung der Oxalsäure und einiger ihrer Derivate auf die Nieren der Versuchsthiere hatte.

I. Versuche mit Oxalsäure.

Die Versuche mit Oxalsäure wurden an einer Reihe von Kaninchen und Hunden angestellt. Die Oxalsäure wurde in 
1.

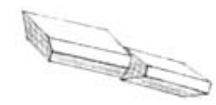

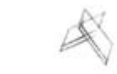

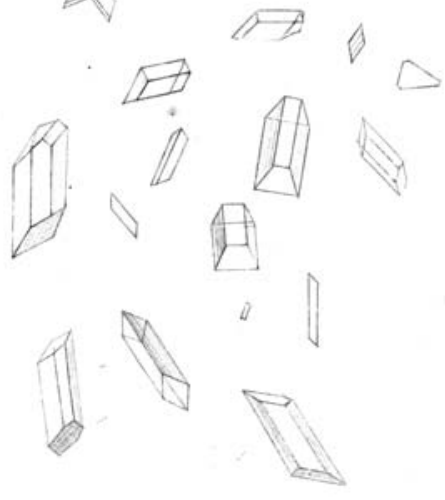

2

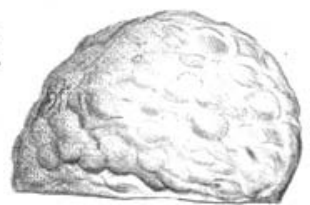

3.

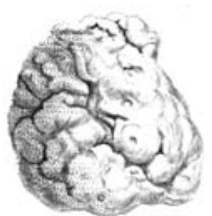

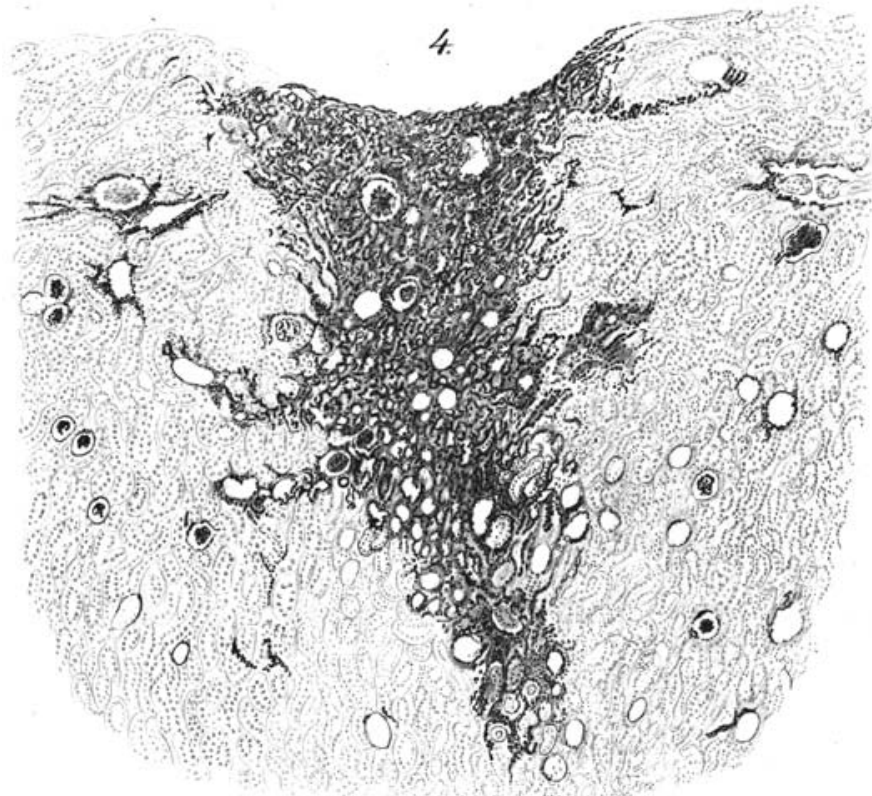

W. (ixphmann sc. 
destillirtem Wasser gelöst per os, bei Kaninchen mit Hülfe der Schlundsonde eingeführt. Die einmal bei einem Hunde versuchte subcutane Injection erwies sich als eine sehr schmerzhafte, wie die Section ergab, von Nekrose an der Injectionsstelle gefolgte Procedur, von der nach zweimaliger Anwendung Abstand genommen warde, insbesondere da nach einer Dosis von $0,8 \mathrm{~g}$ bei dem betreffenden Hunde danach ebenso wie nach der Einverleibung durch den Mund Erbrechen eintrat. Der Zweck unserer Versuche war unserer ursprünglichen Absicht entsprechend nicht der, acut tödtlich verlaufende Vergiftungen za erzengen; die Tagesdosis betrug bei Kaninchen $0,1-0,2 \mathrm{~g}$ Oxalsäure, und die Versuche erstreckten sich auf etwa eine Woche. Bei Hunden wurde die Einverleibung der Oxalsâure länger, bei einem Hunde 18 Tage lang fortgesetzt, und zwar wurden an je 9 Tagen 0,4 , bezw. $0,8 \mathrm{~g}$ Oxalsäure, im Ganzen $10,8 \mathrm{~g}$ gegeben. Der betrefende Hund zeigte am Tage vor seinem Tode taumelnden Gang and Schwäche auf den Hinterbeinen.

Betreffs der Symptome, welche die Versuchsthiere darboten, haben wir unsere Aufmerksamkeit besonders dem Harn zugewendet. Derselbe reagirte bei verschiedenen Versuchskaninchen verschieden, bei einem Thiere war er sauer, bei den anderen meist alkalisch. Eiweiss fehtte in Harn nicht, Harncylinder wurden theils in grōsserer Menge gefunden, theils aber völlig vermisst. Auch die mit dem Harn ausgeschiedenen Krystalle von Calciumoxalat batten eine verschiedene Form, indem sie in einigen Fällen Briefconvertin anderen Hantelform zeigten.

Bei einem Hunde fanden wir den Orin sauer, er enthielt eine sehr geringe Nenge Eiweiss, wäbrend Cylinder und Calciumoxalatkrystalle fehlten. Bei einem anderen Hunde waren im Harn mässige Mengen Eiweiss und viel Calciumoxalatkrystalle, sowie einmal ausgelaugte rothe Bluthörperchen vorhanden.

Was die nach subacuter und chronischer Oxalsäurevergiftung im Harn gelegentlich auftretende, mannichfach umstrittene, stark reducirende Substanz betrift, so ist bekannt, ' däss Kobert in dieser Beziehung widersprechende Mittheilungen gemacht bat, inden er einmal in einer mit $K u$ ssner gemeinschaftlich veröffentlichten Arbeit ${ }^{1}$ ) angiebt, dass es sich dabei um einen optisch tnactiven und nicht vergäbrbaren Körper handele, während or an anderem Orte ${ }^{2}$ mittheilt, dass diese reducirende Substanz die Polarisations-

1) Kobert und Küssner, Zeitschrift für die gesammten Naturwissenscbaften. 3. Folge. Bd.4. S. 101. Berlin 1879 und dieses Archiv. Bd. 78. 8. 234. 1879.

э) Kobert, Zur Pharmakologie der Oxalsănre und ihrer Derivate, Ver- 


\section{8}

ebene drehe und vergährbar sei: Wir baben notirt, dass der Harn zweier Versuchskaninehen bei Darreichnng von 0,1 , bezw. $0,2 \mathrm{~g}$ Oxalsăure während 7 , bezw. 8 Tagen kaum and bei einem Hunde, der innerbalb 18 Tagen mit dem Futter $10,8 \mathrm{~g}$ Oxalsänre, und zwar an je 9 Tagen 0,4 , bezw. $0,8 \mathrm{~g}$ Oxalsäure erbielt, gar nicht Feblingsche Lösung reducirte. Bei einem anderen Hunde, dew innerbalb 136 Tagen $69,7 \mathrm{~g}$ Oxalsäure in Dosen von $0,2-0,7 \mathrm{~g}$ einverleibt worden war und dessen Urin mässige Mengen Eiweiss enthielt, reducirte derselbe Febling'sche Lösung. Die gleiche Urinbeschaffenbeit fand sich bei einem Hunde, der während 162 Tagen $101,3 \mathrm{~g}$ Oxâlsäure in Dosen von $0,2-0,7 \mathrm{~g}$ und $326 \mathrm{~g}$ Chlorcalcines in Gaben von $1,5-3 \mathrm{~g}$ bekommen batte. Auch bei einem bleinen Hunde, der im Laufe von 30 Tagen mit $257 \mathrm{~g}$ oxalsaurem Kalk (die grösste Tagesdosis 10, die kleinste 5 g) gefüttert war, reducirte der alkalische, bleine Menge Eiweiss enthaltende Harn, in dem meist Calciumoxalatkrystalle fehlten, Febling'sche Lösung. Wir konnten aber nie das Ausfallen von Kupferoxydul beobachten.

Bei der Section fanden sich bei fast allen Kaninchen schon makroskopisch sichtbare Veränderungen der Nieren, nehmlich auf der Oberfäche und auf dem Durchschnitt del Rinde und des Markes zahl'eiche weissgrane Pünktchen. Auch bei einem Hunde, dem $18 \mathrm{Tage} \mathrm{lang,} \mathrm{und} \mathrm{zwar} \mathrm{an} \mathrm{je}$ 9 Tagen 0,4 und $0,8 \mathrm{~g}$ Oxalsäure gegeben warde, konnten wir sie auf der Oberfiäche und dem Durchschnitte der Niere, deren Kapsel schwer abziebbar war, beobachten, besonders reichlich fanden sie sich in einer $0,5 \mathrm{~cm}$ breiten Zone zwischen der Binde und der Marksubstanz. In den Nieren des Hundes, der in 136 Tagen mit $69,7 \mathrm{~g}$ Oxalsäure gefüttert war, fehlten diese makroskopiscb sichtbaren, weissgrauen Pünktchen an der Grenze von Mark und Rinde, und waren nur vereinzelt in der Rinde und der Marksubstanz vorbanden.

Die mikroskopische Untersuchung der Nieren der Hunde und Kaninchen ergab, dass die mikroskopisch sichtbaren grauweissen Pünktchen aus in Salzsäure Jöslichen, in Essigsäure aber unlöslichen, das Licht. doppelt brechenden Krystallen bestanden, die das Lumen mebr oder minder zahtreicher Harnkanälchen der Rinde, weniger båufger des Markes, ausfüllten, und die wir als Krystalle von oxalsaurem Kalk angesprochen baben. Sie waren meist farblos, seltener hatten sie einen gelblichen Farbenton und zeigten verschiedene Formen. Neben Sphärokrystallen, die nicht nur zum Theil eine concentrische Schichtung, sondern auch eine radialfaserige Streifung zeigten, sahen wir sanduhr- und semmelförmige Krystalle und ferner aus nadelähnlichen Krystallen bestehende keilförmige Aggregate, die vielfach wit ibren Spitzen an einander gelagert waren, so dass doppelkeilähnliche Figuren entstanden; zuweilen fanden wir auch kurze Säulen wit spitzen oder abgerundeten Fnden. Diese Krystallablagerungen haben wir nur bei dem mit oxalsaurem Kalk gefütterten Hunde vermisst. Diese, die Harn-

handlungen der Gesellschaft deutseher Naturforscher und Aerzte zu Halle 1891. Theil II. S. 198. Leipzig 1892. 
kanälchen infarcirenden Krystalle konnten wir mit saurem Hämatoxylin (Ehrlich) färben und haben weiter die Beobachtung gemacht, dass nach Behandlung mit verdünnter Salzsäure von diesen Krystallen ein ihrer Form und Grösse entsprechendes Gerüst zurückbleibt. Das Epithel der Harnkanälchen, in denen diese Krystalle lagen, war theils verändert, theils war es durch sie zusammengedrückt und in einzelnen Kanälchen war es offenbar zu Grunde gegangen. Die Harnkanälchen des Marks und der Rinde, die frei von Krystallablagerungen waren, waren theils intact, eine Reibe in der Rinde gelegene zeigten sich mehr oder weniger stark erweitert und gelegentlich fanden sich Defecte im Epithel und Epithelzellen lagen in ihrem Lumen. Vorzugsweise in der Rindensubstanz fanden sich im interstitiellen Gewebe, aber meist nur spärlich, wenig ausgedehnte Anhäufungen von Rundzellen. Einige inmitten solcher zelligen Infiltrationen gelegene Glomeruli schienen verkleinert zu sein. Im Uebrigen fehlten ausgesprochene Glomerulusveränderungen. In den Glomerulis haben wir nie Ablagerungen von oxalsaurem Kalk gefunden.

\section{Versuche mit Amiden der Oxalsäure.}

Die Oxalsäure bildet bekanntlich zwei Amide, die Oxaminsäure und das Oxamid. Ueber die Wirkung dieser beiden Amide auf den Thierkörper liegt die kurze Mittheilung von Kobert ${ }^{3}$ ) vor, dass sowohl die Oxaminsäure wie das Oxamid sehr giftig seien.

A. Versuche mit Oxaminsäure und ihren Verbindungen.

Der Versuche mit der Oxaminsäure und ihren Verbindungen ist bereits kurz in unserem Buche über die experimentelle Erzeugung von Harnsteinen ${ }^{2}$ ) und in einer Arbeit von L. Oelkers ${ }^{3}$ ) Erwähnung gethan. Diese Mittheilungen scheint Hoffmeister nicht gekannt zu haben, denn er sagt in einer kürzlich erschienenen Arbeit "Ueber die Bildung des Harnstoffes durch Oxydation ${ }^{4}$ ", dass über das Schicksal der Oxaminsäure im Thierkörper nichts Näheres bekannt sei. Er theilt daher an dieser Stelle kurz Versuche von Leo Schwartz mit, die das Ergebniss lieferten, dass die Oxaminsäure im Thierkörper -

1) Kobert, Lehrbuch der Intoxicationen. Stuttgart 1898. S. 742.

2) Wiesbaden 1891. S. 109.

3) L. Oelkers, Ueber Oxaminsäure. Berichte der deutschen chemischen Gesellschaft. Jahrg. XII. 1889. S. 1566.

4) Archiv für experiment. Pathol. und Pharmakol. Bd. 37. S. 440.

A rehiv f. pathol. Anat. Bd. 148. Hft. 2. 
welche Versuchsthiere benutzt wurden, ist nicht gesagt -, soweit sie angegriffen wird, ohne erhebliche vorgängige Oxalatbildung in Harnstoff äbergeht und demgemäss selbst in grossen Gaben ungiftig sei. Bei drei unserer Versuchsthiere haben wir Oxaminsäure, die wir als rein aus einer chemischen Fabrik bezogen, benutzt und sie den Thieren mit dem Futter beigebracht. Der erste Hund bekam innerhalb 8 Tagen bei einer Tagesdosis von $0,5-2 \mathrm{~g}$ in Summa $7 \mathrm{~g}$, der zweite Hund, $9 \frac{1}{2} \mathrm{~kg}$ schwer, bei einer Tagesdosis von 1 in Summa $13 \mathrm{~g}$, der Hund III in 35 Tagen bei einer Tagesdosis von 1,5-3 $\mathrm{g}$ in Summa $57 \mathrm{~g}$.

Die Thiere zeigten dabei keine Krankheitserscheinungen.

Der stets alkalisehe Urin enthielt nur bei Bund III an einem Tage Eiweiss; fast constant aber bei den 3 Hunden Krystalle von oxalsaurem Kalk; bei Hund II reducirte der Urin stark Febling'sche Lösung, doch fiel kein Kupferoxydul aus.

Bei der Section der durch Verbluten getödteten Thiere fand sich bei Hund I ausser einer zooparasitären Tuberculose ') der Lungen und der Nieren eine Verfettung der Epithelien der geraden Harnkanälchen der Nierenrinde, bei Hund III waren auf der Oberfläche und auf dem Durchschnitt der Niere besonders zablreich im Bereich einer 0,1 cm breiten Zone zwisehen Rinde und Mark weissgraue Pünktchen, die, wie die mikroskopische Untersuchung ergab, aus oxalsaurem Kalk bestanden, sichtbar. Die Organe von Hund II boten dagegen einen normalen Befund.

Die mikroskopische Untersuchung von gefärbten Präparaten der Nieren des Hundes I und II bot, wenn wir von den bei Hund I durch die Zooparasiten entstandenen Veränderungen abseben, nichts Abnormes. Dagegen zeigten sich bei Hund III ausser Ablagerungen von oxalsaurem Kalk in den Harnkanälchen der Rinde und des Marks - in der Uebergangsschicht waren sie besonders reichlich - noch Dilatation von Gruppen von Harnkanälchen der Rinde, in denen das Epithel je nach dem Grade der Erweiterung mehr oder weniger stark abgeflacht war, und endlich Blutungen in die Kanäleben des Marks neben starker Füllung der Gefässe des Marks. Gelegentlich baben wir in der Rinde auch Rundzellenanhäufungen geseben, die zu einer Atrophie der Kanălchen gefährt hatten.

Das Auftreten von Calciumoxalatkrystallen im Harn dieser drei Hunde und in den Nieren eines Versuchsthieres (Hund III) legte den Gedanken nahe, ob nicht die von uns benutzte Oxaminsäure unrein, insbesondere oxalsäurebaltig sei.

Eine Prüfung des Schmelzpunktes bestätigte in der That den Verdacht, dass wir mit einem unreinen Präparat gearbeitet

1) Vgl. Ebstein und Nicolaier, Beiträge zur Lehre von der zooparasitären Tuberoulose. Dieses Archiv. Bd. 118. 1889. S, 432. 
hatten, denn der Schmelzpunkt für unsere Oxaminsäure lag zwischen $124-132^{\circ} \mathrm{C}$., während er in den Lehrbüchern der Chemie nach den Angaben von Toussaint auf $173^{\circ} \mathrm{C}$. angegeben wird. Dies veranlasste uns durch den damaligen chemischen Assistenten der Klinik, Dr. L. Oelkers'), reine Oxaminsäure darstellen zu lassen. Die von ihm nach der Methode von Toussaint aus Oxamid dargestellte Säure hatte einen Schmelzpunkt von $210^{\circ} \mathrm{C}$. Nachdem nun Oelkers ausserdem bei der aus oxaminsaurem Kalk dargestellten Oxaminsäure ebenfalls den Schmelzpunkt von $210^{\circ} \mathrm{C}$. feststellte, muss dieser Schmelzpunkt für die reine Oxaminsäure als zutreffend festgehalten werden.

Ein $11 \frac{1}{2} \mathrm{~kg}$ schwerer Hund erhielt von dieser reinen $0 \mathrm{xaminsäure}$ in 10 Tagen $60 \mathrm{~g}$; die Tagesdosis schwankte von $3,5-7 \mathrm{~g}$; das Thier blieb munter und schied einen stark sauren Urin aus. Im Sediment des Harnes fanden sich regelmässig ungefärbte Krystalle, die nach der chemischen Untersuchung aus oxaminsaurem Kalk-bestanden. Die Krystalle liessen sich schon in dem aus der Harnblase ausgedrückten Urin nachweisen und bilden sich also schon im thierischen Organismus. Die Krystalle sind in Salzsăure löslich und wurden beim Behandeln mit Essigsäure streifig. Sie sind auf Taf. V Fig. I abgebildet. Wurde zu dem Urin Chlorcalciumlösung binzugesetzt, so entstand ein weisser Niederschlag, der Kalk enthielt und, wie die Kalkbestimmung ergab, aus oxaminsaurem Kalk bestand. Es gaben nehmlich

$0,6435 \mathrm{~g}$ des lufttrockenen Kalksalzes 0,3042 Calciumsulfat,

oxaminsaurer Kalk enthielt nach Berechnung

d. i. 13,93 pCt. Calcium, 13,89

Der flund wurde durch Verbluten getödtet. Bei der Section fand sich in den Nieren eine zooparasităre Tuberculose, und die mikroskopische Untersuchung derselben zeigte, abgeseben von einer auch normaler Weise gelegentlich vorkommenden Verfettung der Ferrein'schen Pyramiden und den durch die zooparasitäre Tuberculose veranlassten Veränderungen, nichts Abnormes.

Es ergiebt sich also aus diesem Versuch, dass verfütterte reine Oxaminsäure beim Hunde als solche, bezw. als oxaminsaurer Kalk im Urin erscheint. Ob ein Theil der Oxaminsüure in Harnstoff übergeht, wie Leo Schwartz gefunden hat, haben wir nicht untersucht. Ferner lehrt dieser Versuch, dass die Angaben Kobert's, dass die Oxaminsäure sehr giftig sei, für den Hund wenigstens nicht zutreffend ist; denn selbst grössere Dosen einige Zeit verfüttert, wirkten bei unserem Versuchsthiere nicht toxisch, und vermochten auch nicht die Nieren zu schädigen.

1) Oelkers, a. a. 0 . 


\section{2}

Von den Verbindungen der Oxaminsäure wählten wir zu unseren Versuchen: a) das oxaminsaure Ammoniak, b) das Oxamaethan.

a) Versuche mit oxaminsaurem Ammoniak.

Mit dieser Verbindung stellten wir einen Versuch an, über den wir schon früher in unserem Buche ${ }^{1}$ ) berichtet haben. Es sei hier nur bemerkt, dass der zu dem Versuche benutzte Hund in 26 Tagen mit $156 \mathrm{~g}$ reinem oxaminsaurem Ammoniak (Tagesdosis im Mittel $6 \mathrm{~g}$ ) gefüttert warde. Das Thier blieb dabei gesund. Der Urin reagirte stark sauer, er war eiweiss- und zuckerfrei, in seinem Sedimente fanden sich stets Krystalle aus oxaminsaurem Kalk. Auch hier schied sich aus dem Urin nach Zusatz von Chlorcalciumlösung ein aus Krystallen von oxaminsaurem Kalk bestehender Niederschlag aus. Mikroskopisch zeigten die Nieren keine Veränderungen.

b) Versuche mit Oxamaethan.

Das Oxamaethan ist der Aethylester der Oxaminsäure. Unseres Wissens sind Untersuchungen über die Wirkung dieses Körpers auf den thierischen Organismus bisher noch nicht bekannt. Das von uns zu den Versuchen benutzte Präparat hatte einen Schmelzpunkt von $112-113^{\circ} \mathrm{C}$, und es wurden mit ihm an 8 Hunden, 7 Kaninchen, 1 Katze, 3 Ratten und 6 Mäusen Versuche angestellt. Mit Ausnahme der Kaninchen, denen die wässrige Lösung des Oxamaethans mit der Schlundsonde in den Magen gebracht wurde, bekamen die Versuchsthiere das in Wasser gelöste Oxamaethan mit dem Futter gemischt.

Von den 8 H unden wurden gefüttert:

\begin{tabular}{rcccc} 
No. & $\begin{array}{c}\text { Gewicht } \\
\text { in }\end{array}$ & \multicolumn{2}{c}{ mit einer } \\
I. Tagen & kg & \multicolumn{2}{c}{$\begin{array}{c}\text { Tagesdosis Gesammtmenge } \\
\text { von Oxamaethan }\end{array}$} \\
I. & 6 & 2 & $6-7 \mathrm{~g}$ & $13 \mathrm{~g}$ \\
II. & & 3 & $5-10-$ & $15-$ \\
III. & & 16 & $5-$ & $80-$ \\
IV. & 12,5 & 10 & $8-$ & $80-$ \\
V. & & 10 & 10. & $100-$ \\
VI. & & 15 & $5-10-$ & $105-$ \\
VII. & & 14 & $5-10-$ & $135-$ \\
VIII. & 23 & 31 & $2,5-18-$ & $358,5-$
\end{tabular}

i) Ebstein und Nicolaier, Ueber experimentelle Erzengung von Harnsteinen. Wiesbaden 1891. S. 108. 
Alle Thiere blieben munter, doch war der sauer reagirende Urin nur bei 4 Versuchsthieren ( $I$, II, III, VI) frei von Eiweiss, bei Hund V, VII, VIII enthielt er mässige, bei Hund IV reichliche Mengen Eiweiss, Fehling'sche und Nylander'sche Lösung wurden in der Regel nicht reducirt. Einmal trat bei Hund III in dem mit Febling'scher Lösung gekochten Urin eine geringe Ausscheidung von Kupferoxydul auf. Im Urinsediment fanden sich bei Hund III in dem eiweissfreien Urin spärliche hyaline Cylinder, bei Hund V ausgelaugte rothe Blutkörperchen, bei sämmtlichen Versuchsthieren waren in dem Sedimente die bereits oben beschriebenen Krystalle von oxaminsaurem Kalk') vorbanden. Beim Versetzen des sauren Urins mit Chlorealeiumlösung schied sich aus dem Urin aller Hunde oxaminsaurer Kalk ${ }^{1}$ ) aus.

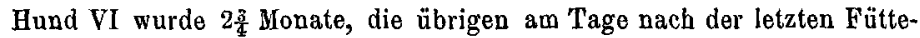
rung mit Oxamaethan getödtet.

Bei der Section dieser 8 Hunde fanden sich in allen Fällen in den Nieren zooparasitäre Tuberculose und die Epithelien der geraden Harnkanälehen der Rinde verfettet. 2 Hunde hatten ausserdem zooparasitäre Knötchen in den Lungen.

Die mikroskopische Untersuchung von gefärbten Schnitten der Nieren zeigte bei sämmtlichen Hunden, abgeseben von den für die zooparasitäre T'uberculose der Hundeniere charakteristischen Veränderungen, stellenweise $z$ wischen den Harnkanälchen der Rinde eine spärliche Menge von Rundzellen, die mit den Tuberkeln nicht in Zusammenhang standen. Bei einem Hunde (V) waren einzelne Gruppen von Kanälchen dor Rinde durch gewuchertes Bindegewebe auseinander gedrängt. Auffällig war an den Epithelien der Harnkanälchen, deren Lumen nicht selten theils hyaline, theils granulirte Cylinder mit gefärbten Zellkernen, theils körnige, nicht gefärbte Massen enthielt, eine Bildung von Vacuolen. Die Färbung der Epithelkerne war eine gute, nur bei Hund $V$ saben wir in der Uebergangsschicht in Gruppen von Kanälchen ausgedehnte Nekrosen des Epithels, so dass die Membrana propria mit einer meist homogenen Zone ausgekleidet war, in der bur ganz vereinzelt noch ein oder mebrere, blass tingirte Kerne sicb fanden. In zwei Fällen zeigten einzelne Glomeruli eine Verdickung ihrer Kapsel, bei 3 Eunden (II, III, V) war eine Verdiekung der Media an einer Reihe von Arterien nachweisbar, bei Hund $V$ erwies sich auch an einzelnen grösseren Arterien die Intima gewuchert.

Von den 7 Kaninchen erbielt:

$\begin{array}{rccc}\text { No. } & \text { an Tagen } & \text { eine Tagesdosis eine Gesammtmenge } \\ \text { I. } & 2 & 1,5 \mathrm{~g} & 3 \mathrm{~g} \\ \text { II. } & 2 & 2,5- & 5- \\ \text { III. } & 1 & 5-5 & 5- \\ \text { IV. } & 2 & 2,5- & 5- \\ & \text { (mit } 3 \text { tägiger Pause) } & & \\ \text { V. } & 4 & 1,5- & 6- \\ \text { VI. } & 4 & 2- & 8- \\ \text { VII. } & 7 & 1-2- & 9-\end{array}$

1) Vergl. L. Oelkers, a. a. O. 
Bei Kaninchen IV trat während der Fütterung zunächst Parese, später Paralyse der hinteren Extremitäten ein, bei den übrigen Thieren feblten diese Symptome. Doch enthielt der Urin, der bei 5 Kaninchen (III, IV, V, VI, VII) untersucht wurde, Eiweiss, bei IV und VI in grösserer, bei III in spärlicber Menge, bei $V$ und VII nur an einem Tage, Fehling'sche Lösung reducirte er beim Kochen nicht. Im Sediment waren stets Krystalle von oxaminsaurem Kalk, bei Kaninchen III, IV, VI Cylinder, theils hyaline, die häufig mit Epithelzellen besetzt waren, theils granulirte.

Mit Ausnahme von Kaninchen VI, welches getödtet wurde, gingen alle Versuchsthiere in Folge der Fütterung mit Oxamaethan zu Grunde. Die Nieren der 7 Kaninchen zeigten bei der Section eine mehr oder weniger stark ausgesprochene Hyperämie.

Bei Kaninchen V fanden sich auf der Oberfäche der linken Niere Blutungen, ferner auf der Magenschleimbaut hämorrhagische Erosionen, bei Kanincben VI liess die Magenschleimbaut ausser starker Hyperämie stellenweise Nekrosen, zum Theil auch kleine Geschwäre erkennen.

Die mikroskopisebo Untersuchung ergab bei Kaninehen V und VI Veränderungen in der Magenschleimbaut, dio den makroskopisch sichtbaren (Hyperămie, bämorrbagische Erosionen, Nekrosen und Geschwüren) entsprachen; in der Leber fanden sich bei Kaninchen III und IV in dem interacinösen Gewebe spärliche Mengen von Rundzellen und bei Kaninchen V war das interacinöse Bindegewebe stark verbreitert, zum Theil auch in die Leberläppchen bineingewuchert.

Bei sämmtlichen Kaninchen zeigten die Nieren pathologische Veränderungen, die bei den einzelnen Thieren gleichartig, jedoch nicht immer gleich stark ausgesprochen waren. Die Intensität der Veränderungen entsprach keineswegs immer der verfütterten Menge Oxamaethan, bezw. der Dauer des Versuches. In gefärbten Präparaten der Nieren fanden sich Anhäufungen von Rundzellen vorzugsweise in der Rinde zwischen den Harnkanälchen, um die Glomeruli und häufig auch um die Gefässe. Diese Rundzellenanhäufungen batten meist keine sehr grosse Ausdehnung, so dass die Harnkanälehen, $z$ wischen denen sie lagen, vicht geschädigt waren. Nur bei Kaninchen I, III und VII waren sie stellenweise in der Rinde so umfänglich, dass die in ihnen gelegenen Kanälchen atrophisch geworden waren. $\mathrm{Zu}$ einer Wucherung des interstitiellen Bindegewebes war es in keinem Falle gekommen. Die Epithelien der Harnkanälchen zeigten durchweg gute Kernfärbung, doch waren sie in manchen Kanälchen ausser Reib und Glied gekommen und lagen regellos in ihrem Lumen. Häufig war das Lumen der Harnkanälchen mit einer granulirten, nicht tingirten Masse erfällt, violfach lagen in ihm besonders in den Kanälchen der Rinde hyaline, granulirte und Epithelialcylinder; in einzelnen dieser Cylinder enthaltenden Kanälchen feblte das Epitbel. In Lumen einzelner Harnkanälchen fanden wir auch rothe Blutkörperchen. Blutungen, meist nicht sehr ausgedehnt, zwischen den Harnkanälchen und in den Glomeruluskapseln waren nicht selten. Die Gefässe waren bei allen Thieren mehr oder weniger stark mit Blut gefüllt, 
insbesondere war dies bei Kaninchen II, III, IV stark ausgeprägt. Bei zwei Kaninchen (II und VII) waren die Kerne der Glomeruli auffillig vermebrt; nur einmal, bei Kaninchen II, saben wir die Glameruli arm an Kernen, so dass die Gefässe vielfach als hyaline Schlingen erschienen.

Eine Katze wurde an 10 Tagen mit $32 \mathrm{~g}$ Oxamaethan gefüttert. Die Tagesdesis schwankte $z$ wischen 2,5 und $5 \mathrm{~g}$. Wabrend der Versuchsdauer schied das Thier mit dem Urin im Mittel 1 pro mille Eiweiss aus (Bestimmung mit dem Esbach'schen Albuminimeter), Zucker enthielt der Urin vicht. Im Sediment wurden neben zahlreichen Krystallen von oxaminsaurem Kalk sehr zahlreiche granulirte Cylinder gefunden.

Die Section des durch Verbluten getödteten Thieres orgab eine zooparasitäre Tuberculose der Lungen und Nieren und eine sebr starke Verfettung der Nierenrinde. Die Untersuchung von gefärbten Präparaten der Niere zeigte ausser den durcb Zooparasiten verursachten Tuberkeln vielfach zwischen den Harnkanälchon der Rinde meist spärliche Mengen von Rundzellen und in den Kanälchen der Rinde und des Markes Cylinder, die meist byalin waren.

Die drei zu den Versuchen benutzten Ratten gingen nach Fütterung mit Oxamaethan zu Grunde. Bei Ratte I, die einmal 0,5 g Oxamaethan bekommen hatte, ergaben sich weder makroskopisch noch mikroskopisch Organveränderungen. Dagegen waren bei Ratt $\mathrm{II}$, die in 5 Tagen mit $4 \mathrm{~g}$ (Tagesdosis $0,5-1,0$ ) und bei Ratte III, die in 11 Tagen mit $10 \mathrm{~g}$ (Tagesdosis $0,25-2,0)$ gefüttert wurde, Nieren und Leber, bei Ratte II auch die Milz, stark byperämisch und bei Ratte III liessen sich in der Magensebleimhaut zablreiche hämorrhagische Erosionen, im Mageninhalt viel rothe Blutkörperchen nachweisen. Auch bei diesen Thieren fanden sich Urin-Erystalle von oxaminsaurem Kalk. Bei den letzten beiden Ratten waren in den Nieren Verfettungen der Epithelien vorhanden, die indess nicht in gleicher Intensität und Extensität; bei Ratte II fanden sich diese Veränderungen nur an den Epitbelien der gewundenen Kanälchen der Rinde und waren geringgradig, bei Ratte III erstreckten sie sich auch auf die Harnkanälchen des Marks und waren sehr stark ausgeprägt. Gefärbte Schnitte der Niere von Ratte II und III zeigten ausser starker Gefassfüllung, vereinzelten Cylindern in den Harnkanälcben der Rinde unả ganz spärlichen Rundzellenanbăufungen im interstitiellen Gewebe der Rinde keine pathologiscbe Verändernngen.

Bei 6 Mäusen, bei denen die Darreichung von kleinen Oxamaetbandosen tödtlich wirkte, haben wir in den Organen, insbesondere in den Nieren, patbologische Veränderungen vermisst. Im Urin der Mäuse fanden wir stets Krystalle von oxaminsaurem Kalk.

Fassen wir das Resultat der vorstehenden Versuche kurz zusammen, so ergiebt sich Folgendes: Nach Fütterung mit Oxamaethan trat bei allen unseren Versuchsthieren oxaminsaurer Kalk in dem Urin auf und bei Hunden, bei denen wir darauf- 
hin untersucht haben, fand sich im Harn ausserdem freie Oxaminsäure. Bei sämmtlichen Thieren - nur die Mäuse machten eine Ausnahme - waren mehr oder weniger stark ausgeprägte Veränderungen in den Nieren vorhanden. Während Hunde grosse Dosen bis $15 \mathrm{~g}$, selbst wenn diese einige Zeit gegeben wurden, ohne Störung ihres Befindens vertrugen und ebenso eine Katze nach mehrtägiger Fütterung von $2,5-5 \mathrm{~g}$ ohne Krankheitserscheinungen blieb, gingen Kaninchen und Ratten, nachdem die Fütterung kleiner Dosen (bei Kaninchen $1-2,5 \mathrm{~g}$, bei Ratten $0,5-1,0 \mathrm{~g}$ ) mehrere Tage lang fortgesetat war, zu Grunde. Auch Mäuse starben bald wach Darreichung kleiner Dosen.

\section{B. Versuche mit Oxamid.}

a) Einverleibung des Oxamids durch Fütterung.

Ueber die Fütterungsversuche mit Oxamid haben wir bereits kurz auf dem 8. Congress für innere Medicin zu Wiesbaden $\left.{ }^{12}\right)$, sowie in der 5 . Abtheilung des 10 . internationalen medicinischen Congresses $z a$ Berlin $^{1 b}$ ) und ausführlich in unserem Buche ${ }^{10}$ ) berichtet. Das Resultat dieser Versuche lässt sich dahin zusammenfassen, dass es gelingt, durch Fütterung grösserer Dosen Oxamid bei einer Reihe von Thierspecies insbesondere boi Hunden, Kaninchen und Katzen in relativ kurzer Zeit Harnsteine, bei denen der sog. Steinbildner das Oxamid ist, in den verschiedenen Theilen des Harpapparates zu erzeugen. Krohl ${ }^{2}$ ), der unter Leitung von Kobert arbeitete, hat nach

2) a) Verbandlungen des 8 . Congresses für innere Medicin zu Wiesbaden. 1889. S. 268.

b) Verbandlungen des 10. internationalen medicinischen Congresses zu Berlin. 1890. Bd. II. 5. Abtheilung. S. 37. Bei beiden Gelegenheiten wurden die Oxamidconcremente selbst, ihr Aufbau an Dünnsehliffen und an Präparaten ibres organiscben Gerüstes, sowie die Veränderungen der Harnorgane an makroskopischen und mikroskopischen Präparaten und Photogrammen erläutert. Ausserdem bat Herr Gebeimrath König diese Präparate dem 18. Congresse der Deutschen Gesellschaft für Chirurgie in Berlin 1889 vorgelegt.

c) Ueber die experimentelle Erzengung von Harnsteinen. Wiesbaden 1891 .

2) Krobl, Paul, Zur Kenntniss der Wirkungen der Oxalsäure und einiger Derivate derselben. Arbeiten des pharmakologischen Instituts $\mathrm{zu}$ Dorpat, berausgegeben von R. Kobert. Bd. VII. 1891. S. 147 ff. 
Veröftentlichung unserer beiden ersten Mitheilungen diese Versuche wiederholt und konnte unsere Versuchsergebnisse vollkommen bestätigen. Wir möchten darauf besonders hinweisen, weil nehmlich Kobert und Küssner ${ }^{1}$ ) früher bei Fütterungsversuchen mit Oxamid zu ganz anderen Resultaten gekommen sind. Kobert und Küssner hatten nehmlich damals gefunden, dass durch Fütterung von Säugethieren mit Oxamid eine chronische Vergiftung herbeigeführt wird, die derjenigen gleicht, welche im Gefolge von Natriumoxalat entsteht, sowohl was die Symptome während des Lebens, als auch, was die nach dem Tode constatirten Organveränderungen der Versuchsthiere anlangt. Namentlich fanden die Autoren das Verhalten der Niere so wie bei der Vergiftung mit Natriumoxalat; von Concrementbildung haben sie nichts erwähnt. Kobert und Küssner nehmen auf Grund dieser Versuche an, dass das Oxamid im Thierkörper, wenn nicht ganz, so doch zum Theil in Oxalsäure übergeht, und dass es dadurch im Stande sei, eine chronische Oxalsäurevergiftung hervorzurufen. Wir hatten in unserem Buche über die experimentelle Erzeugung der Harnsteine ${ }^{2}$ ) diese abweichenden Versuchsergebnisse von Kobert und Küssner zum Theil wenigstens aus der Verschiedenheit bezw. aus der Unreinheit (Verunreinigung mit Oxalsäure) des von Kobert und Küssuer benutzten Oxamids, über dessen Eigenschaften sich keine Angaben bei ihnev vorfinden, erklärt. Neuerdings bat Kobert ${ }^{3}$ )

1) Kobert und Küssner, Zeitschr. für die gesammten Naturwissensch. 3. Folge. Bd. 4. S. 101. Berlin 1879 und Dieses Archiv. Bd. 78. 1879. S. 242.

2) a. a. O. S. 18 .

3) Kobert theilt in diesem Bericht mit, dass seine Versuche bereits abgeschlossen waren, als unser Buch über die experimentelle Erzeugung von Harnsteinen erschien. Er citirt bei dieser Gelegenheit diese unsere ausführliche Mittheilung und unterlässt es, unsere erste Veröffentlichung aus dem Jahre 1889, die zwar einen kurzen, aber doch vollständigen Bericht unserer Studien über Oxamidsteinbildung giebt, zu erwähnen. Wir möchten dies besonders hervorbeben, weil es nach dem Bericht Kobert's scheinen könnte, als sei die Thatsache, dass bei gewissen Thieren nach Fütterung mit Oxamid Harnsteine entstehen, von Kobert und uns fast gleichzeitig gefunden und nur von uns etwas früherveröfentlicht worden. In der That sind aber, wie aus der später er- 
in einem Bericht über die Versuche Krohl's in der XIII. Abtheilung der 64 . Versammlung deutscher Naturforscher und Aerzte zu Halle a. S. ${ }^{1}$ ) die Mittheilung gemacht, dass das von ihm damals vor 13 Jahren angewandte Oxamid rein gewesen sei und führt als Beweis an, dass es neutral reagirte, schöne Krystalle bildete und selbst bei längerem Contact mit Wasser nichts in Lösung gehen liess. Wir können indess nicht anerkennen, dass auf diese Weise der Beweis für die Reinheit des Oxamids geliefert ist; der sichere Nachweis, dass Oxamid rein ist, kann nur, wie das auch von uns gemacht ist, nur durch eine Elementaranalyse geliefert werden. Gesetzt aber, das von Kobert und Küssner damals angewandte Oxamid sei rein gewesen, so haben die Autoren, da sie besonders hervorheben, dass sie stets bei ihren Versuchsthieren das Verhalten der Niere so wie bei der Vergiftung mit Natriumoxalat fanden, den Irrthum begangen, dass sie die Ablagerungen im Nierengewebe, die bei den Thieren nach Fütterung mit Oxamid auftraten, für Ablagerungen von oxalsaurem Kalk, wie sie nach Darreichung von Oxalsäure, bezw. deren Salzen in den Harnkanälchen der Rinde sich vorfinden, gehalten haben. Dieser Irrthum ist um so schwerer verständlich, als sich schon makroskopisch die Oxamidablagerungen durch ihre gelbe Farbe von den weissgrauen Calciumoxalatablagerungen unterscheiden, insbesondere aber bei der mikroskopischen Untersuchung der Unterschied beider sofort in die Augen fällt.

$\mathrm{Krohl}{ }^{2}$ ) hat die Beobachtung gemacht, dass bei einigen

schienenen ausführlichen Arbeit von Krobl hervorgeht, die Versuche Krohl's, über die Kobert damals berichtet hat, erst nach dem Erscheinen unserer ersten Mittheilung aus dem Jahre 1889 begonnen und durch sie veranlasst worden. Unsere boiden ersten Veröffentlichungen mussten übrigens Kobert bekannt sein, erstens da wir sie in unserem, von ihm citirten Buche über experimentelle Erzeugung von Harnsteinen S. 16 erwähnt haben und zweitens, weil Krobl, der unter seiner Leitung arbeitete, unserer ersten Veröffentlichung Erwähnung gethan hat. Auch in seinem sehr eitatreichen Lehrbuche der Intoxicationen hat Kobert gelegentlich der Besprechung der Oxamidsteinbildung weder unsere ausführliche Mittheilung, noch unsere frühere Veröffentlichung citirt.

1) Verhandlungen der Gesellschaft deutscher Naturforscher und Aerzte zu Halle 1891. Theil II. S. 198. Leipzig 1892.

2) a. a. 0 . 
mit Oxamid gefütterten Thieren Glykosurie auftrat. Wir konnten, wie unsere Versuchsprotocolle ergeben, und wie wir in unserem Buche S. 69 besonders hervorgehoben haben, nie bei unseren Hunden Zucker im Urin nachweisen.

Krohl hat ferner gefunden, dass das Oxamid bei trächtigen Thieren in kleinen Dosen mehrmals täglich ( 3 mal täglich $1 \mathrm{~g}$ ) Abort hervorruft. Wir bezweifeln nicht, dass ein so differenter Körper wie das Oxamid, welches insbesondere die Harnorgane schwer zu schädigen vermag, zuweilen auch bei trächtigen Thieren za Abort Veranlassung geben kann. Dass dies aber keineswegs immer der Fall ist, beweist der in unserem Buche ${ }^{1}$ ) angeführte Versuch VIII. Derselbe betraf eine trächtige Hündin, die an 22 Tagen mit 101,5 g Oxamid (die Tagesdosis schwankte zwischen $2-8 \mathrm{~g}$ and betrug im Mittel $4,2 \mathrm{~g}$ ) gefüttert wurde. Ein Abort trat nicht ein. Nachdem die Fütterung eine Zeit lang ausgesetzt war, warf das Thier zwei gesunde, ausgetragene Junge, die von ihr gesäugt wurden und sich gut entwickelten.

Eine weitere Bestätigung haben übrigens unsere Versuchsergebnisse betreffend die experimentelle Erzeugung von Harnsteinen durch Oxamidfütterung von Seiten Tuffier's ${ }^{2}$ ), Navarro's ${ }^{3}$ ) und de Rouville's ${ }^{4}$ ) erhalten.

Wir wollen an dieser Stelle noch kurz über Versuche bei Kaninchen und einem Hunde berichten, die wir nach vorgängiger subcutaner Injection mit neutralem chromsanrem Kali mit Oxamid fütterten. Das neutrale chromsaure Kali erzeugt, wenn es den Versuchsthieren in genügend grossen Dosen gegeben wird, ausgedehnte Epithelnekrosen in der Niere, und

I) a. a. 0. S. 38.

2) Tuffier, Lithiase urinaire artificielle. Bulletins de société anatomique de Paris. 1893. p. 49. - Lithiase urinaire expérimentale d'après la méthode d'Ebstein et Nicolaier. Archives de physiologie normale et pathologique. 5. série. T.5. 1893.

3) Navarro, Lithiase renale expérimentale avec hydronephrose. Bulletins de la société anatomique de Paris. 1893. p. 658.

4) de Rouville, Lithiase urinaire expérimentale d'après la méthode d'Ebstein et Nicolaier. Nouveau Montpellier méd. IIt. p. 473. 1894. Citirt aach Virchow-Hirsch's Jahresbericht. 1894. 
wir haben diese Versuche in der Absicht angestellt, um zu erfahren, ob auch bei Versuchsthieren, bei denen das Nierengewebe stark geschädigt war, die Fütterung von Oxamid Concremente in den Harnwegen erzeugt.

Wir experimentirten an drei Kaninchen und einem Hunde. Zwei Kaninchen (I und II) war vor der Oxamidfütterung $0,1 \mathrm{~g}$ neutrales ebromsaures Kali in wässriger Lösung subcutan injicirt. Es trat in Folge davon am zweiten Versuchstage im Urin, dessen Menge erbeblich vermindert war, reichlich Eiweiss auf; am dritten Versuchstage wurde dann das Oxamid gefüttert, und zwar bei beiden Kaninehen je $5 \mathrm{~g}$. Das Kanineben I liess bis zu seinem am 5 . Versuchstage durch ein Trauma erfolgenden Tod keinen Urin, bei dem Kaninchen II fanden sich am 5. Versuchstage in dem immer noch eiweissreichen, in spärlicher Menge abgesonderten Urin Oxamidkrystalle. Bis zum 6. Versuchstage, an dem das Thier starb, wurde kein Urin mehr gelassen.

Bei der Section zeigten die Nieren beider Thiere auf der Oberfläche eine Reihe gelbbräunlicher und gelber Stellen, und bei Kaninchen II waren ausserdem noch flache, narbenähnliche Einsenkungen vorhanden. Oxamidconcremente wurden in den Harnwegen nicht gefunden.

Das Mikroskop zeigte bei beiden Thieren in Sehnitten der frischen Nieren sehr reichlich Fett in den Epithelien der Harnkanälchen der Rinde und in gefärbten Präparaten der gehärteten Niere sehr zablreich Nekrosen der Epithelien, vorzugsweise der gewundenen Harnkanälchen, ferner hyaline und granulirte Cylinder und gelegentlich Blutungen in die Kanälchen des Markes und der Rinde und kernarme Glomeruli, die übrigens recht gross erschienen. Ausserdem fanden wir spärliche Mengen von Rundzellen zwischen den Harnkanälchen und vielfach anch um die Gefässe. Bei Kaninchen II waren entsprechend der an der Oberfliche der Nieren makroskopisch sichtbaren Vertiefung die Harnkanälchen grösstentheils durch ausgedehnte Anhäufung von Rundzellen, die sich von der Oberfläche bis in das Mark hineinerstreckten und mebrfach die Form eines Keiles hatten, dessen Basis nach der Oberfläche zugekehrt war, zu Grunde gegangen. Die im Bereich dieser Rundzellenanhäufungen liegenden Glomeruli waren atrophisch. Ablagerungen von Oxamidkrystallen haben wir in den Präparaten nieht gesehen.

Bei Kaninchen III hatten wir die Versuchsanordnung derart abgeändert, dass wir zunächst $0,05 \mathrm{~g}$ neutrales chromsaures Kali subcutan injicirten, dann am 2. und 3. Versuchstage mit 5 , bezw. $2,5 \mathrm{~g}$ Oxamid fütterten. Am 3. Versuchstage wurde ausserdem $0,075 \mathrm{~g}$ neutrales chromsaures Kali eingespritzt und am 4. Tage 2,5, am 5. und 6. Tage je $3 \mathrm{~g}$ Oxamid per os einverleibt. Am 6. Versuchstage trat der Tod ein. Das Kaninchen hatte also im Ganzen 0,125 $\mathrm{g}$ neutrales chromsaures Kali und $16 \mathrm{~g}$ 0xamid erhalten. Auch bei diesem Kaninchen war der Urin schon am 2. Versuchs- 
tage eiweisshaltig, seine Menge war indess reichlich, Oxamidkrystalle fehlten in ibm. Seit dem 4. Versuchstage bestand Anurie, die bis zum Tode fortdauerte.

Bei der Section erwies sich die Oberfläche der Niere, die stellenweise gelb gefärbt war, glatt. Auch hier fanden sich keine Oxamidconcremente in den Harnwegen. Der mikroskopiscbe Befund stimmte im Wesentlichen mit dem beim Kaninchen 1 orhobenen äberein.

Ein junger, etwa $\frac{1}{4} \mathrm{Jahr}$ alter $\mathrm{Hund}$, dem am 1. und 5. Versuchstage $0,05 \mathrm{~g}$ neutrales chromsaures Kali subcutan injicirt wurde, erbielt mit der Schlundsonde am 2.-4. Versuchstage 2,5, 3,0 und 2,5 g. Der Urin konnte nicht untersucht werden. Tod am 5. Tage. Abgesehen von einer stellenweise gelben Farbe ihrer Oberfläche boten die Nieren makroskopisch nichts Abnormes. Keine Oxamidconcremente.

Abgesehen von Epithelnekrosen in den Harnkanälchen der Rinde waren keine pathologischen Veränderungen zu constatiren. Oxamidablagerungen wurden auch hier vermisst.

Die Versuche an Kaninchen zeigen also, dass verfüttertes Oxamid auch von den durch die Wirkung des neutralen chromsauren Kali schwer geschädigten Nieren ausgeschieden wird, dass aber keine Concremente sich gebildet haben. Wir glauben indess, dass es möglich ist, auch unter diesen Verhältnissen Concremente durch Oxamidfütterung zu erzeugen, wenn es gelingt, die Fütterungsversuche längere Zeit, als es uns bei unseren Versuchsthieren möglich war, fortzusetzen.

b) Subcutane Einverleibung des Oxamids.

Wir hatten bereits in unserem Buche ${ }^{1}$ ) über Versuche bei einer Maus und bei einem Kaninchen berichtet, welche beweisen, dass das Oxamid bei subcutaner Application von den Lymphbahnen aus aufgenommen wird und im Harn zur Ausscheidung gelangt. Wir haben nun noch weitere Versuche mit der subcutanen Einverleibung des Oxamid angestellt, um die Frage zu entscheiden, ob auch auf diese Weise bei Thieren sich Harnsteine erzeugen lassen.

Wir experimentirten zunächst mit lKaninchen. Es wurde diesen Thieren Oxamid (einmal $7 \mathrm{~g}$ ) unter die Haut eingebracht, bezw. in Paraffinum liquidum suspendirt subcutan eingespritzt. Entzündliche Veränderungen wurden danach an den Applicationsstellen nicht beobachtet; in denjenigen

1) a. a. 0. S. 198 . 
Fällen, wo etwas grössere Mengen Oxamid eingebracht wurden, bildete sich im subcutanen Gewebe eine knotenförmige Verdickung, die wohl grösstentheils durch Oxamid bedingt wurde. Diese Verdickung war in dem Falle, wo $7 \mathrm{~g}$ Oxamid subcutan eingebracht worden waren, noch 3 Wochen nach der Einverleibung vorbanden. Bei diesen Kaninchen fanden sich nach etwa 2-3 Tagen im Urin Oxamidkrystalle in Form von Sphärolithen, welche bei Untersuchung mit dem Polarisationsmikroskop das Interferenzkreuz gaben. Diese Ausscheidung dauerte eine Zeit lang an, indessen konnten wir bei der Section dieser Thiere Concremente in den Harnorganen nicht nachweisen.

Günstiger waren die Versuchsresultate bei Hunden. Das Oxamid wurde ihnen in derselben Weise wie den Kaninchen suboutan eingebracht. An den Applicationsstellen traten bei diesen Thieren starke Druckempfindlichkeit und eine ergiebige Ansammlung von seröser Flüssigkeit ein, die in mebreren Fällen sich nach aussen entleerte. 6-8 Tage nach Einbringung des Oxamids wurden meist in dem zuweilen eiweissbaltigen Urin Krystalle von Oxamid, häufig auch in Form von Sphärolitben gefunden. Bei den Hunden, die getödtet wurden, fanden sich in den Harnorganen im Nierenbecken und in der Blase grössere und kleinere Oxamidconcremente. Wir sehen indessen nur diejenigen Fälle für die Entscheidung der Frage, ab subertane Application von Oxamid Harnsteine erzeugen kann, für beweisend an, bei denen eine Entleerung der subcutan gebildeten Flüssigkeit nach aussen nicht stattgefunden hat, weil wir nur bei diesen Fällen die Möglichkeit für ausgeschlossen halten, dass das Oxamid durch Ablecken der Oxamid enthaltenden Flüssigkeit von den Thieren per os aufgenommen wurde.

Wir fügen hier kurz einen dieser Versuche an. Einem Teckel wurde $5 \mathrm{~g}$ Oxamid, das in Wasser zu einem dicken Brei angerübrt war, in eine Hanttasche gebracht, und die Haut durch Nähte gescblossen. Etwa 6 Tage nachher war die Wunde geheilt. In ibrer Umgebung fand sich eine hühnereigrosse, fluctuirende Geschwulst. Am 8. Tage liessen sich in dem mässig eiweisshaltigen Urin spärliche rothe und ausgelangte Blutkörperchen und zablroiohe Oxamidlurytalle, die aueh am 10. Versuchstage noch vorbanden waren, nachweisen. Am 11. Versuchstage wurde der Hund getôd tet.

Bei der Section zeigten nur die Harnorgane pathologische Veränderungen. In beiden Nierenbecken fanden sich mehrere kaum linsengrosse, in der Harnblase eine Reibe bis hanfkorngrosser, gelber Oxamidconcremente mit klein warziger Oberfläche. Der Durchsebnitt der Niere zoigte in der Marksubstanz vereinzelte gelbe Streifehen, die aus Oxamidablagerungen bestanden. Dio mikroskapische Untersuchung der frischen Nieren ergab eine Verfettung der Epithelien der geraden und auch in geringem Grade der der gewundenen Harnkanälchen. Die Haut war an der Stelle, wo das Oxamid eingebracht war, durch eine Narbe geschlossen. 
c) Versuche mit Oxamid und Oxalsäure.

Wir haben früher ${ }^{1}$ ) beobachtet, dass bei einem Hunde nach Fütterung mit grösseren Dosen Oxamid, das durch Oxalsäure verunreinigt war, im Nierenbecken und in der Harnblase Concremente sich gebildet hatten, die aus Oxamid und oxalsaurem Kalk bestanden. In einem anderen Versuche ${ }^{2}$ ) ist uns bei Fütterung mit grossen Dosen reinem Oxamid, dem wir, um wieder Oxalsäure-Oxamidsteine experimentell zu erzeugen, kleine Dosen Oxalsäure zusetzten, dies nicht gelungen. Wir erhielten Concremente, welche lediglich Oxamid als Steinbildner enthielten. Nach einer Reihe analoger Versuche änderten wir die Versuchsanordnung derart, dass wir Hunde längere Zeit mit kleinen Dosen von Oxamid und Oxalsäure fütterten, zunächst in der Hoffnung, dass es uns gelingen würde, wieder oxalsäurehaltige Steine auf experimentellem Wege zu erzeugen. In dieser Beziehung schlugen dio Versuche fehl. Sie führten nicht zur Concrementbildung, ergaben aber insofern ein bemerkenswerthes Resultat, als bei einigen dieser Versuchsthiere sich Schrumpfnieren entwickelten, welche wir auf dem 11. Congresse für innere Medicin ${ }^{3}$ ) demonstrirt haben.

Die drei, 7-11 kg schweren Hunde, bei denen diese Nierenverănderungen beobachtet wurden, bekamen mit sebr wonigen Ausnahmen täglich $0,3 \mathrm{~g}$ 0xamid und $0,6 \mathrm{~g}$ 0xalsäure mit dem Futter, und zwar wurden

Hund I während 169 Tagen mit 50,7 g Oxamid und 101,4 g Oxalsäure gefüttert.

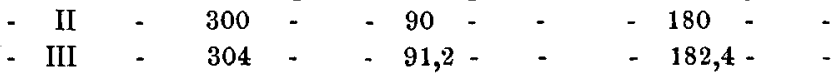

Die Thiere blieben während der ganzen Versuchszeit munter; bei allen dreien enthielt der meist sauer reagirende Urin Eiweiss, fast stets in geringer Menge, reducirte aber Fehling'sche Lösung nicht. Im Sediment waren bei Hund I in den letzten Monaten der Versucbszeit hyaline, bei Hund III epitheliale Cylinder in spärlicher Menge vorhanden, bei Hund II fehlten sie,

1) Ebstein und Nicolaier, Ueber experimentelle Erzeugung von Harnsteinen. Wiesbaden 1891. S. 36. (Versuch VII.)

2) a. a. 0. S. 42. (Versuch IX.)

3) Ebstein und Nicolaier, Ueber experimentelle Erzengung von Schrumpfnieren durch Oxalsäure-0xamidfütterung. Verhandl. des 11. Congr. für innere Med. Wiesbaden 1892. S. 518. 
dagegen fanden sich bei Hund II ebenso wie bei Hund I gegen Ende des Versuches ausgelaugte rothe Blutkörperchen. Oxamidkrystalle wurden stets vermisst; Calciumoxalatkrystalle wurden häufig, jedocb nicbt constant beobacbtet.

Bei der Section der drei Hunde, die durch Verbluten getödtet wurden, zeigten sich vorzugsweise nur die Nieren verändert, und zwar boten sie bei Hund I und III das typische Bild der Schrumpfnieren (Taf. V. Fig. 2 und 3, von denen Fig. 2 nach einem in Müller'scher Flüssigkeit und in absoluten Alkohol, No. 3 nach einem gekochten uud dann in Alkohol conservirten Stück einer Niere vom Hupde I gezeichnet ist). Es fanden sich an den verkleinerten Nieren, deren Kapsel leicht abziebbar war, Partien mit kleinhöckeriger Oberfläche, die man an einzelnen Stellen geradezu als granulirt bezeichnen konnte, auch eine grosse Zahl vertiefter, eingezogener Stellen, über die das normale, bezw. wenig veränderte Parenchym hervorragte. An den gekochten Nieren trat die Einziehung der Oberflãche noch erbeblich deutlicher hervor. Ausserdem waren an der Oberfläche der Nieren eine Reihe bis über hirsekorngrosser, mit einer hellen Flüssigkeit gefüllter Cysten sichtbar. Die normalen Stellen zeigten im Wesentlichen die Farbe der Hundeniere, wäbrend die gescbrumpften Partien eine graue Farbe hatten. Auf dem Durchschnitt liessen sich in dem normal gefärbten, bräunlichrothen Parenchym der Rinde graue, meist strangförmige mit den eingezogenen Partien der Oberfäche in Zusammenhang stehende und gelbe, im Bereich der geraden Barnkanälchen gelegene Stellen unterscheiden. Die Marksubstanz batte auf dem Durchschnitt eine grawweisse Farbe, und bei Hund I fanden sich in der Spitze der Papille beider Nieren graugelbliche, kleine körnchenartige Einlagerungen; beim Hund III waren diese Einlagerungen nur in der linken Niere vorhanden.

Beim Hund III ergab die Messung der Nieren folgende Zablen:

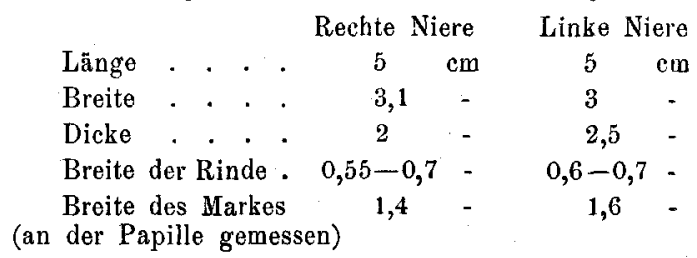

Beim Hund II boten die Nieren ein ähnliches Bild, nur waren die Veränderungen derselben nicht so weit vorgeschritten, wie bei den Nieren der beiden anderen Hunde. Die eingesunkenen grauen Stellen der Oberflāche, die, wie der Durchschnitt der Niere zeigte, sich anch mehr oder weniger tief in die Rinde fortsetzten, waren weniger zahlreich, es fehlte eine ausgesprochene Körnung der Oberfläche, und auch Cysten wurden nicht beobachtet. Im Mark fanden sich auch hier punkt- und strichförmige Ablagerungen. Das Herz zeigte bei den drei Hunden keine Veränderungen. Die Dicke seiner Musculatur betrug auf dem Durchschnitt 


\section{5}

$\begin{array}{lcc} & \text { des rechten Ventrikels } & \text { des linken Ventrikels } \\ \text { beim Hund I } & 0,7 & 1,0 \\ \text { beim Hund II } & 0,35 & 1,1 \text { dickste Stelle } \\ & & 0,35 \text { dünnste - }\end{array}$

Die frischen Nieren zeigten mikroskopiscb starke Verfettungen, die beim Hund I die Epithelien der geraden Harnkanälchen betrafen, während beim Hund III ausserdem die. Epithelien der gewundenen Kanälchen und der Harnkanälchen des Markes, wenn auch nur in geringem Grade, verfettet waren. Bei allen drei Hunden liessen sich auch Ablagerungen von Calciumoxalat in den Harnkanälchen der Rinde, vielfach auch in denen des Markes nachweisen.

Die mikroskopische Untersuchung gefärbter Schnitte der in Alkohol gehärteten Nieren ergab bei allen 3 Hunden insofern eine Uebereinstimmung, als wir bei ihnen ganz gleichartige Veränderungen fanden, freilich waren sie nicht bei allon dreien gleich stark entwickelt und zeigten auch nicht die gleiche Ausdehnung; bei Hund III waren sie am wenigsten ausgebildet. Wir fassen die Beschreibung des mikroskopischen Befundes bei den 3 Versuchshunden kurz zusammen (Taf. V Fig. 4).

Entsprechend den vertieften eingezogenen Stellen der Nieren fanden wir eine ausserordentlich starke Wucherung des Bindegewebes, das mehr oder weniger grosse Mengen von Rundzellen zeigte. Diese Bindegewebswucherungen, welche sich meist in Form eines Keiles, dessen Basis der etwas verdickten Nierenkapsel anlag, mehr oder weniger tief in die Rinde erstreckten, grenzten sich nicht immer ganz scharf gegen das normale, bezw. weniger veränderte Gewebe $a b$, und waren schon bei makroskopischer Besichtigung der Schnitte durch ihre stärkere Tinction deutlich kenntlich. Innerhalb dieses gewucherten Bindegewebes war der grösste Theil der Harnkanälchen zu Grunde gegangen. Die wenigen, die noch vorhanden waren, zeigten sich vielfach atrophisch und bäufig war ihr sehr enges Lumen mit hyalinen $\mathrm{Cy}$ lindern oder Calciumoxalatkrystallen erfüllt. Auch die Zahl der Glomeruli war im Bereich dieser Bindegewebskeile sehr stark reducirt, sie lagen in Folge der Schrumpfung des Gewebes hänfig sehr nahe an einander und erwiesen sich nur selten unverändert. Wir konnten an den Glomeruluskapseln und den in ihnen enthaltenen Schlingen und zwar an den einzelnen Glomerulis in verschiedener Entwickelung, Ausdehnung und Combination alle die mannichfachen Prozesse nachweisen, die als charakteristisch für die Glomerulonephritis des Mensechen gehalten werden. Eine Umwandlung der Glomeruli in byaline Klümpchen, die so oft bei analogen Prozessen in der menschlichen Niere gefunden werden, haben wir indess nicht beobachtet. Einmal haben wir auch zwischen den Glomerulusschlingen krystallinische Ablagerungen beobachtet, die nicht nur in der Form, sondern auch in ihrem Verhalten Lösungsmitteln gegenüber (Löslichkeit in Salzsäure, Unlöslichkeit in Essigsäure) den in den Harnkanälchen gefundenen glichen und die wir, wie diese, als Krystalle von Calciumoxalat angesprochen baben. An

Archiv f. pathol. Anat. Bd. 148. Hft. 2. 
den nicht eingesunkenen Stellen der Rinde fanden sich insbesondere in der Nähe der Bindegewebskeile mehr oder weniger ansgedehnte Anhäufungen von Rundzellen und gelegentlich auch Wucherung des interstitiellen Bindegewebes. Auch an diesen Partien waren an den Harnkanälchen und an den Glomerulis die gleichen Veränderungen in verschiedener Ausdebnung und Entwickelung vorhanden, wie wir sie an den in den geschilderten Heerden gelegenen beobachtet baben. Vielfach erschienen hier die Glomerulusveränderungen hochgradiger als die der Harnkanälchen. In den Harnkanälchen des Markes konnten wir auch zuweilen Cylinder und Calciumoxalatkrystalle nacbweisen. Ausgesprochene Gefässveränderungen haben wir nicht gesehen. Es mag hier noch bemerkt werden, dass wir in diesen Nieren Oxamidablagerungen eben so wenig wie Concremento geseben baben. Bei zwei Huaden, die wir gleichfalls lăngere Zeit mit kleinen Dosen Oxamid

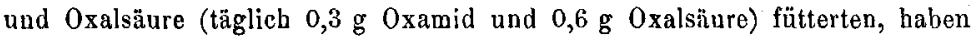
wir Schrumpfnieren nicht gefunden. Von diesen beiden Hunden erhielt

der erste (VI) während 295 Tagen $88,5 \mathrm{~g}$ Oxamid und $177 \mathrm{~g}$ 0xalsäure - zweite (V) - $363-108,9-\quad-\quad 217,8$ -

Auch diese Thiere blieben während der Versuchszeit munter, bei Hund $V$ fand sich im Urin Eiweiss in geringer Menge und im Sediment spärliche C ciumoxalatkrystalle, Cylinder fehlten; bei Hund IV war der Urin eiweissfrei und enthielt weder Cylinder noch Krystalle.

Die Section der beiden durch Verbluten getödteten Thiere ergab, dass die Nieren nicht verkleinert, und ihre Oberfläche glatt war. In der Rinde fanden sich bei beiden Hunden zooparasitäre Tuberkel.

Mikroskopisch wurden, abgesehen von den durch die Zooparasiten velanlassten Veränderungen, stellenweise geringe Mengen von Rundzellen im interstitiellen Gewebe der Rinde und in vereinzelten Kanälcben der Rinde Ablagerungen von Calciumoxalat beobachtet.

Wir haben in unserer ersten Mittheilung ${ }^{1}$ ), in der wir über die Versuche bei zwei Hunden (I und III) berichtet haben, bereits darauf hingewiesen, dass wir die Entstehung der Schrumpfnieren als eine Wirkung der Fütterung mit kleinen Dosen Oxamid und Oxalsäure ansehen, dass es sich in diesen Fällen um eine toxische chronische Nephritis, bei der wir alle die Niere zusammensetzenden Gewebe verändert fanden. Obwohl weitere Versuche bei 3 Hunden ergaben, dass nur bei einem (II) Schrumpfnieren sich entwickelten, während bei den beiden jüngeren, trotzdem der eine (IV) in nahezu der gleichen Zeit die gleiche Menge Oxamid und Oxalsäure wie Hund II, der andere (V) bei län-

1) a. a. 0. 
gerer Versuchsdauer grössere Mengen Oxamid und Oxalsäure als Hund III erhielt, dieselben fehlten, so haben wir doch an unserer Ansicht festgehalten, und uns das abweichende Versuchsergebniss so gedeutet, dass die Nieren jüngerer Hunde widerstandfähiger seien gegenüber einer Intoxication von kleinen Dosen Oxamid und Oxalsäure. Wir wurden zu dieser Deutung um so mehr veranlasst, weil wir bei zahlreichen Sectionen von Hunden nie Veränderungen in den Nieren beobachtet haben, die den Sehrumpfungsprozessen in den Nieren unserer 3 Versuchshunde entsprachen, und insbesondere, weil wir in der uns zu Gebote stehenden Literatur über die Nierenerkrankungen des Hundes keine Angaben darüber fanden, dass bei Hunden gelegentlich Schrumpfnieren vorkommen. Neuerdings sind wir indess auf eine Arbeit von $\mathrm{Rabe}^{\mathbf{1}}$ ) aufmerksam geworden, in der er über einen Fall von Nephritis parenchymatosa bei einem 7jährigen Hühnerhunde berichtet wird. Bei diesem Thiere fanden sich in den Nieren Veränderungen, die makroskopisch sowohl wie mikroskopisch mit den bei unseren Versuchsthieren eine grosse Uebereinstimmung zeigten. Herr Prof. Rabe hat uns überdies vor einiger Zeit persönlich mitgetheilt, dass nach seinen Erfahrungen bei älteren Hunden hin und wieder Schrumpfnieren vorkämen, und er hatte die Güte, uns Schrumpfnieren von 2 Hunden, die er gelegentlich gefunden hatte, zur Untersuchung zu überlassen. Die Nieren beider Thiere (bei dem einen war wäbrend des Lebens Eiweiss im Urin, Anasarca und Ascites beobachtet worden) zeigten makroskopisch und mikroskopisch analoge Befunde, wie bei unseren Versuchshunden. Uebrigens wird auch das gelegentliche Vorkommen von Schrumpfnieren bei Hunden durch neuere Arbeiten von Porcher ${ }^{2}$ ) und Liénaux ${ }^{3}$ ) bestätigt.

Mit Rücksicht auf diese Beobachtungen halten wir, da unsere Versuche an zwei jüngeren Hunden ein anderes Ergebniss

1) Rabe, Beitrag zur Kenntaiss der Nierenkrankheiten. Lüneburg 1883.

$\left.{ }^{2}\right)$ Porcher, Ch., Du rein sénile chez le chien. Archives de médecine expérimentale. 1895 . T. 7. p. 488 .

3) Liénaux, Plusieurs cas de nephritis de Bright chez le chien. Annal. belg. 43. Jahrg. XLII. p. 65. Citirt nach Ellenberger und Schütz, Jahresberichte über die Leistungen auf dem Gebiete der Veterinärmedicin. 1894. 
lieferten, die Möglichkeit nicht für ausgeschlossen, dass bei den drei älteren, bei denen wir nach der Fütterung mit Oxalsäure und Oxamid Schrumpfnieren fanden, sich diese auch aus anderen Gründen entwickelt haben und möchten daher die Frage, ob die Fütterung von kleinen Dosen Oxalsäure und Oxamid Schrumpfnieren erzeugt, jetzt nicht in der positiven Weise beantworten, wie wir es früher gethan haben.

\section{Erklärung der Abbildungen.}

Tafel V.

Die Abbildungen sind von Herrn Universitätszeichenlebrer Peters in Göttingen gezeichnet.

Fig. 1. Krystalle von oxaminsaurem Kalk (S. 371). Mittelstarke Vergrösserung.

Fig. 2 und 3. Schrumpfniere vom Hunde (S. 384). Natürliche Grösse.

Fig. 4. Mit Pikrocarmin gefärbter Schnitt durch die Rinde einer Schrumpfniere vom Hunde (S. 385). Schwache Vergrösserung. 